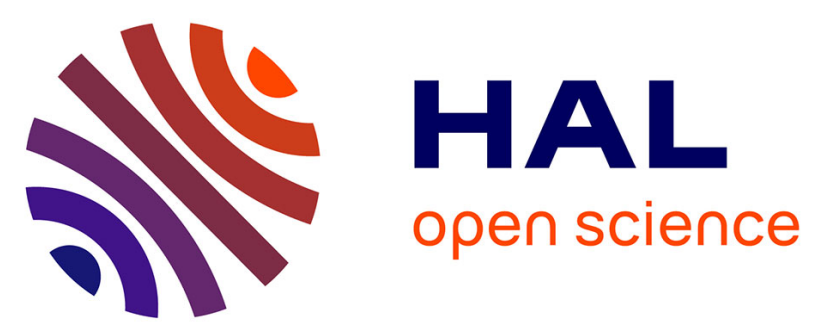

\title{
Verification of a Monte Carlo dose calculation engine in proton minibeam radiotherapy in a passive scattering beamline for preclinical trials
}

Consuelo Guardiola, Ludovic de Marzi, Yolanda Prezado

\section{To cite this version:}

Consuelo Guardiola, Ludovic de Marzi, Yolanda Prezado. Verification of a Monte Carlo dose calculation engine in proton minibeam radiotherapy in a passive scattering beamline for preclinical trials. Brit.J.Radiol., 2020, 93 (1107), pp.20190578. 10.1259/bjr.20190578 . hal-02504664

\section{HAL Id: hal-02504664 \\ https://hal.science/hal-02504664}

Submitted on 20 Apr 2020

HAL is a multi-disciplinary open access archive for the deposit and dissemination of scientific research documents, whether they are published or not. The documents may come from teaching and research institutions in France or abroad, or from public or private research centers.
L'archive ouverte pluridisciplinaire HAL, est destinée au dépôt et à la diffusion de documents scientifiques de niveau recherche, publiés ou non, émanant des établissements d'enseignement et de recherche français ou étrangers, des laboratoires publics ou privés. 
archives-ouvertes

\title{
Verification of a Monte carlo dose calculation engine in proton minibeam radiotherapy in a passive scattering beamline for preclinical trials
}

\author{
Consuelo Guardiola, Yolanda Prezado, Ludovic de Marzi, Kathryn Held
}

\section{To cite this version:}

Consuelo Guardiola, Yolanda Prezado, Ludovic de Marzi, Kathryn Held. Verification of a Monte carlo dose calculation engine in proton minibeam radiotherapy in a passive scattering beamline for preclinical trials. British Journal of Radiology, British Institute of Radiology, In press. hal-02475971

\section{HAL Id: hal-02475971 \\ https://hal.archives-ouvertes.fr/hal-02475971}

Submitted on 12 Feb 2020

HAL is a multi-disciplinary open access archive for the deposit and dissemination of scientific research documents, whether they are published or not. The documents may come from teaching and research institutions in France or abroad, or from public or private research centers.
L'archive ouverte pluridisciplinaire HAL, est destinée au dépôt et à la diffusion de documents scientifiques de niveau recherche, publiés ou non, émanant des établissements d'enseignement et de recherche français ou étrangers, des laboratoires publics ou privés. 


\title{
Verification of a Monte Carlo dose calculation engine in proton minibeam radiotherapy in a passive scattering beamline for preclinical trials
}

\author{
'CONSUELO GUARDIOLA, 'YOLANDA PREZADO, 'LUDOVIC DE MARZI and ${ }^{2}$ KATHRYN HELD \\ ${ }^{1}$ Imagerie et Modélisation en Neurobiologie et Cancérologie, Centre National de la Recherche Scientifique, Marie Curie fellow, Orsay, \\ France \\ ${ }^{2}$ Massachusetts General Hospital, Boston, USA

Objectives: Proton minibeam radiation therapy ( $\mathrm{pMBRT}$ ) is a novel therapeutic strategy that combines the benefits of proton therapy with the remarkable normal tissue preservation observed with the use of submillimetric spatially fractionated beams. This promising technique has been implemented at the Institut Curie-Proton therapy centre (ICPO) using a first prototype of a multislit collimator. The purpose of this work was to develop a Monte Carlo-based dose calculation engine to reliably guide preclinical studies at ICPO.

Methods: The whole "Y1"-passive beamline at the ICPO, including PMBRT implementation, was modelled using the Monte Carlo GATE v. 7.0 code. A clinically relevant proton energy (100 MeV) was used as starting point. Minibeam generation by means of the brass collimator used in the first experiments was modelled. A virtual source was modelled at the exit of the beamline nozzle and outcomes were compared with dosimetric measurements performed with EBT3 gafchromic films and a diamond detector in water. Dose distributions were recorded in a water phantom and in rat $\mathrm{CT}$ images (7-week-old male Fischer rats).

Results: The dose calculation engine was benchmarked against experimental data and was then used to assess dose distributions in CT images of a rat, resulting from different irradiation configurations used in several experiments. It reduced computational time by an order of magnitude. This allows us to speed up simulations for in vivo trials, where we obtained peak-to-valley dose ratios of $1.20 \pm 0.05$ and $6.1 \pm 0.2$ for proton minibeam irradiations targeting the tumour and crossing the rat head. Tumour eradication was observed in the 67 and $22 \%$ of the animals treated respectively.

Conclusion: A Monte Carlo dose calculation engine for PMBRT implementation with mechanical collimation has been developed. This tool can be used to guide and interpret the results of in vivo trials.

Advances in knowledge: This is the first Monte Carlo dose engine for PMBRT that is being used to guide preclinical trials in a clinical proton therapy centre.

\section{INTRODUCTION}

Proton minibeam radiation therapy (pMBRT) is a novel therapeutic strategy ${ }^{1}$ in which irradiation is performed by using arrays of narrow $(\leq 1 \mathrm{~mm})$ proton beams. pMBRT has already been implemented in our clinical centre (Institut Curie-Proton Therapy Center in Orsay, ICPO). ${ }^{2,3}$ The distinct dose delivery method used in pMBRT results in a net reduction in skin damage $e^{4,5}$ and neurotoxicity compared to conventional proton therapy. ${ }^{4}$ Moreover, superior tumour control compared to that obtained with standard proton therapy has been demonstrated in small animal experiments. ${ }^{6}$ Significant tumour control was also observed even with highly heterogeneous dose distributions, ${ }^{7}$ suggesting the participation of distinct biological mechanisms compared to standard RT. Comprehensive biological experiments are needed to elucidate the underlying mechanisms in pMBRT. For this purpose, a suitable set of dosimetry tools in order to accurately guide and interpret preclinical trials at ICPO needs to be developed. The very small beam sizes used in pMBRT makes this a challenging and error-prone task, requiring thorough evaluation (by Monte Carlo simulations and experimental measurements).

In particular, the development of a validated Monte Carlobased dose calculation engine for small animal experiments was necessary to obtain precise and detailed information about the dose distributions actually delivered in the rodent anatomy. As proof-of-concept, we assessed the dose distributions in water and CT images of a rat's head. To 
Figure 1. Schematic view of the Y1-beamline with all beam modifiers. ${ }^{16}$

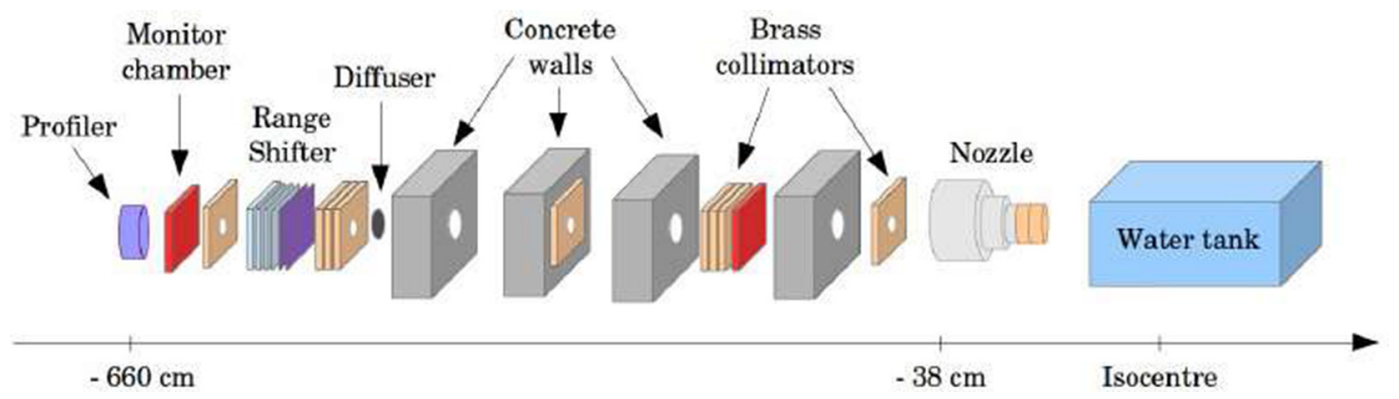

the best of our knowledge, this is the first complete dosimetric study, including a dose calculation engine, for preclinical trials of pMBRT.

\section{METHODS}

\section{Monte Carlo simulations}

Monte Carlo simulations based on the GATE v. $7.0 \operatorname{code}^{8}$ were used to model the Y1-room beamline of ICPO, the multislit collimator, and to assess the dose distributions in the target. GATE v. 7.0 is based on the Geant 4 toolkit (Geant4.9.6.p03). ${ }^{9-11}$ The physics lists and parameters recommended by the GATE collaboration for proton therapy applications were used. ${ }^{12-14}$ In particular, the Binary Cascade (BIC) model was used for the hadronic interactions adding the standard option 3 to include the lowenergy electromagnetic processes (_EMY). Both options were included by using the QGSP_BIC_HP_EMY GATE builder. Range cuts of 1 and $0.025 \mathrm{~mm}$ were considered for all particles for the world and phantoms, respectively. Ionisation potentials for water and air were set to 75 and $85.7 \mathrm{eV}$, respectively. ${ }^{14,15}$ It is worth noting that these GATE recommendations include the use of $75 \mathrm{eV}$ for ionisation potential of the water, which was the recommended value in earlier ICRU reports. However, the newer ICRU 73 recommends an I value of $78 \pm 2 \mathrm{eV}$. For the sake of clarification, we run simulations with the virtual source (Virtual source modelling) with both 75 and $78 \mathrm{eV}$ water ionisation potentials and no discernible differences in the percentage depth dose (PDD) were found in both cases for a $100 \mathrm{MeV}$ proton energy. Consequently, we performed simulations following the GATE collaboration recommendation afterwards for this particular scenario.

\section{Simulation of one passive scattering beamline at ICPO}

In a first phase, the complete beamline was modelled and benchmarked against experimental data. In a second phase, for sake of computational time, a virtual source model was defined by means of an iterative process.

The ICPO facility is equipped with an isochronous cyclotron, a C230 from Ion Beam Applications company, that delivers 230 $\mathrm{MeV}$ protons at the accelerator exit. The treatment room chosen for our first studies was the Y1-room, which has a horizontal fixed beamline and uses a double scattering delivery system. Figure 1 shows a schematic view of the simulated Y1-room components. Further details can be found in Patriarca et al. ${ }^{17}$

Since the main targets of pMBRT are neurological lesions, i.e. brain tumours, and in order to consider the worst treatment scenario (centre of the human brain), we used $100 \mathrm{MeV}$ Bragg peaks as starting-point, which corresponds to a proton range of $7.7 \mathrm{~cm}$ in water equivalent. As the first in vivo experiments of pMBRT focused on rodent brains with tumour diameters less than $5 \mathrm{~mm}$, we used only simple Bragg peaks, which cover 5 and $3 \mathrm{~mm}$ with 95 and $98 \%$ of the prescription (Bragg peak) dose, respectively.

A virtual source was modelled to minimise computational time. For this purpose, beam characteristics at the nozzle exit, i.e. beam energy spread, spot size, and angular distribution, were stored in Phase Space Files, as a function of the beam energy required at

Figure 2. PMBRT brass multislit collimators comprising $3.5 \mathrm{~cm}$ long slits (left) and $2 \times 2 \mathrm{~cm}^{2}$ broad brass collimator (right). To achieve an irradiation area behind the collimator exit, an additional squared-shape brass collimator $\left(1.6 \times 1.6 \mathrm{~cm}^{2}\right)$ was added to the beamline nozzle (right). Image from Cécile. ${ }^{16}$ pMBRT, proton minibeam radiation therapy.

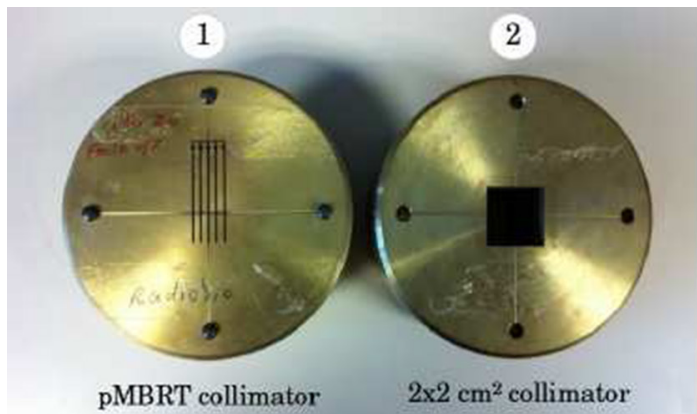

pMBRT collimator

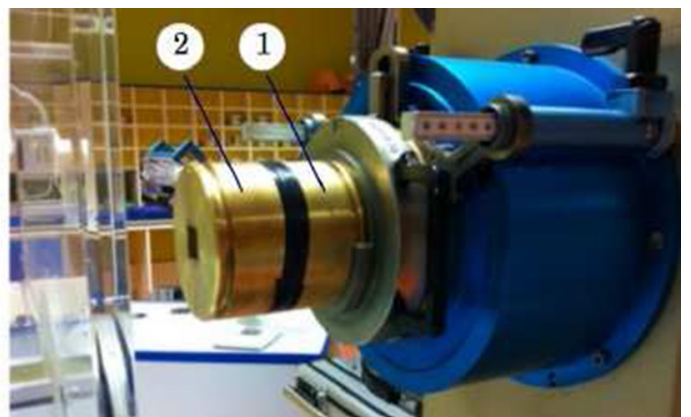


Figure 3. Experimental PVDR values obtained with the PTW diamond detector and EBT3 gafchromic films when a $0.7 \mathrm{~mm}$ wide brass collimator with ctc of $3.5 \mathrm{~mm}$ was used to generate minibeams. PVDR, peak-to-valley dose ratio.

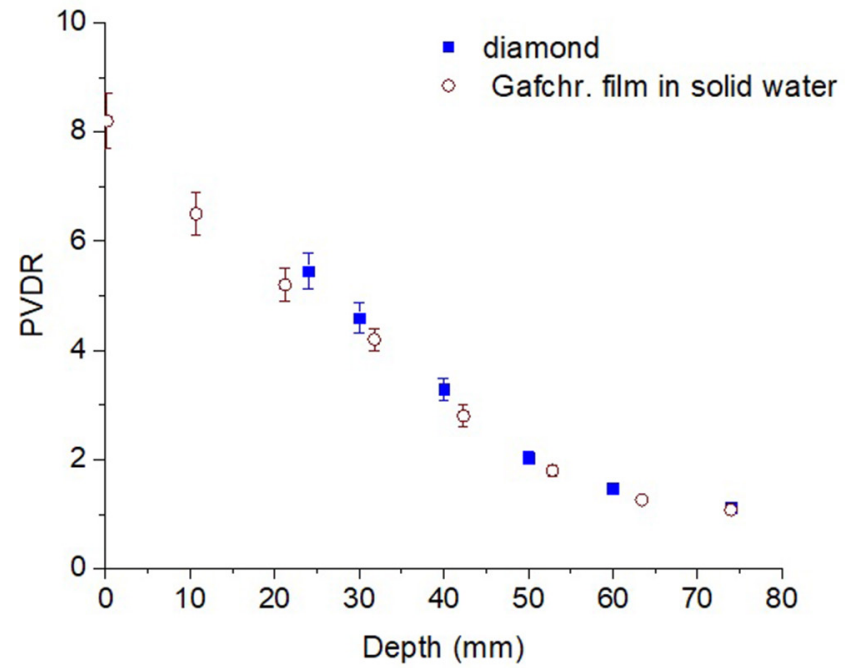

the isocentre. They were used along with the data coming from previous modelling of the same beamline at the Orsay proton therapy centre ${ }^{18}$ as starting point. In particular, a $100 \mathrm{MeV}$ proton energy beam, $1.5 \mathrm{MeV}$ energy spread and $15 \mathrm{~mm}$ spot size was used. The range of angular spread covered ranged from 0.1 to $5 \mathrm{mrad}$ in our iterative process. Those included the typical range of values from 2 to $5 \mathrm{mrad}$ for passive systems. ${ }^{19,20}$ An iterative comparison of simulated and measured dose distributions was performed for fine tuning of the parameters of the virtual source.

A gain of one order of magnitude in computational efficiency was achieved with this approach.

\section{Multislit collimators}

Multislit brass collimators described in the work by Peucelle et $\mathrm{al}^{2,16}$ were used for minibeam generation. These collimators are $5 \mathrm{~cm}$ thick (like the standard used for proton therapy at that energy range), with widths of 0.4 and $0.7 \mathrm{~mm}$ and centre-tocentre (ctc) distances of 3.2 and $3.5 \mathrm{~mm}$, respectively. This optimised design ${ }^{21}$ was manufactured at ICPO by electrical discharge machining (Figure 2).

\section{Dose calculations}

The phantom multislit collimator distance was always $7 \mathrm{~cm}$. Dose distributions were recorded using voxel sizes of $2 \mathrm{~mm} \times 100 \mu \mathrm{m}$ $\times 1 \mathrm{~mm}$ in the vertical, lateral and beam directions, using the so-called GATE-DoseActor. PDD curve, lateral dose profiles, and peak-to-valley dose ratios (PVDRs) were evaluated. The PVDR assessment was performed by averaging over the tally bin size along the beam transverse axis, i.e. $100 \mu \mathrm{m}$, using the doses in the central peak and its adjacent valley.

The number of simulated primary particles was $10^{12}$, resulting in an uncertainty below $1 \%$ in regions where more than $50 \%$ of the maximum dose was deposited. The total dose was the sum of all units used, calculated by a home-made $C++$ code. Dose uncertainty in a single voxel was computed according to the method described by Chetty et al. ${ }^{22}$

The following phantoms were used: (a) water cylindrical phantoms with a diameter of $20 \mathrm{~cm}$ and a height of $20 \mathrm{~cm}$, simulating a human head $^{23}$; and (b) voxelised CT images of a rat head (7-week-old male Fischer rat), corresponding to the age of the animals usually irradiated in our in vivo experiments. We used CT images obtained at Institut Curie from a representative Fisher rat in the same conditions as those of the irradiated set. DICOM images (CT raw format) must be converted to MetaImage format (.mhd). To save computational time, the rodent CT image was re-sampled with resolutions of $0.25,2$, and $1 \mathrm{~mm}$ in the transverse and longitudinal axes, respectively (with reference to the beamline). Image greyscales were converted into material definitions based on the correspondence between Hounsfield units, i.e. voxel values, and materials. The stoichiometric calibration procedure described in Lansonneur et $\mathrm{al}^{24}$ was used to determine the tissue substitute calibration curves of the CT scanner using a set of materials with known elemental composition and physical density close to those of tissue samples. The output file

Figure 4. Left: PDD curve simulated and measured with EBT3 gafchromic films in solid water. Uncertainties of $8 \%$ were considered for measured data. Right: simulated PVDR values versus experimental data recorded with PTW diamond detector when a $0.7 \mathrm{~mm}$ wide brass collimator with a ctc of $3.5 \mathrm{~mm}$ was used to generate minibeams. PDD, percentage depth dose; PVDR, peak-to-valley dose ratio.
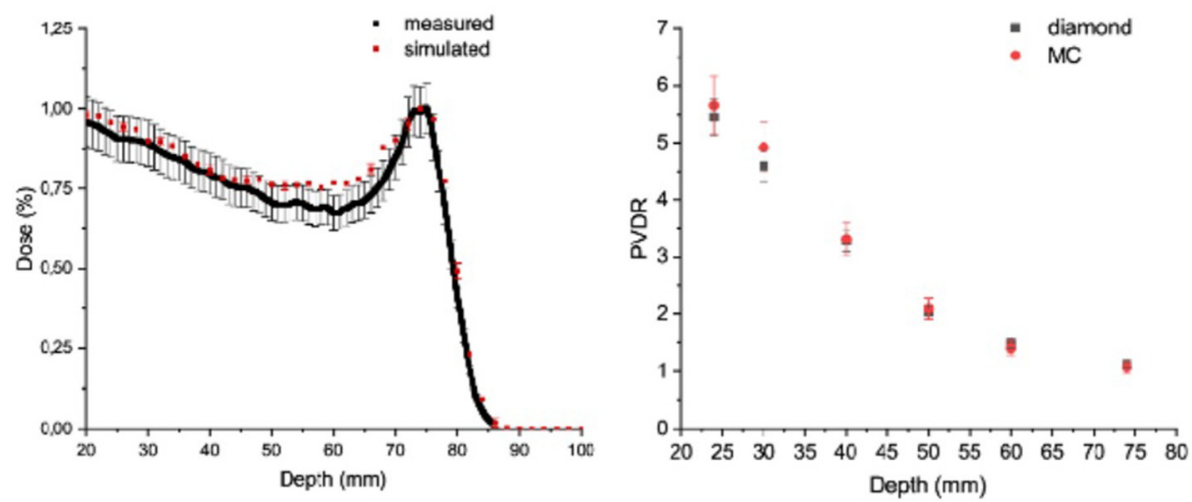
Figure 5. Lateral profile at $24 \mathrm{~mm}$ depth of $\mathrm{MC}$ simulated and experimental data recorded with PTW diamond detector. MC, Monte Carlo.

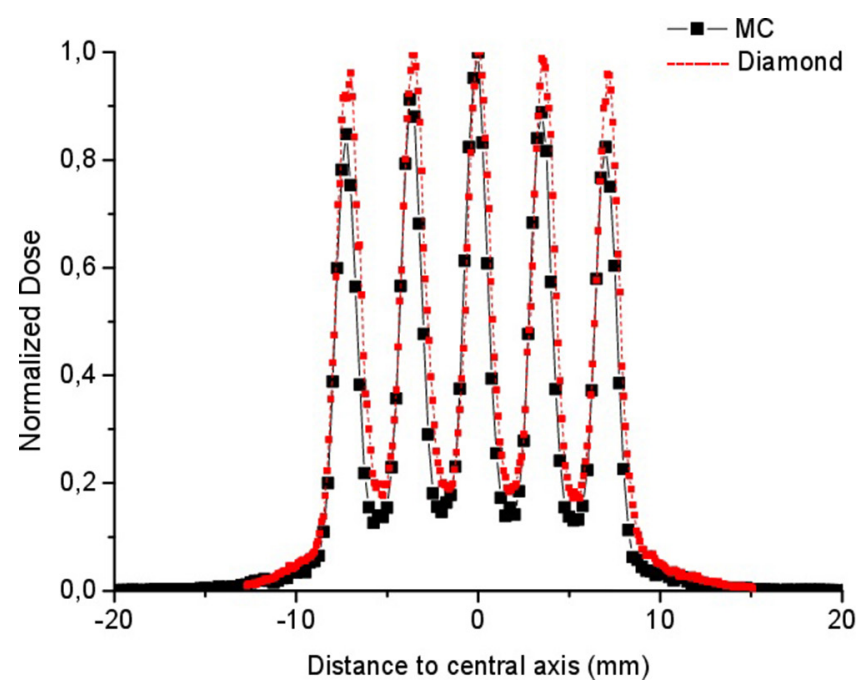

used to collect absorbed doses in the phantom was a binary file containing the absorbed dose in cGy with the same dimensions as the phantom.

Various geometries and energies (50, 70 and $100 \mathrm{MeV}$ ) were considered in the rat brain dose evaluations.

\section{Experimental dosimetry}

Experimental dose distributions were measured with EBT3 gafchromic films ${ }^{25}$ and a PTW microDiamond 60019 detector. ${ }^{26}$ Lateral and depth dose profiles, as well as PVDR values were assessed. Data were then used to validate the dose-calculation engine. Diamond detectors are nearly water equivalent for all beam energies and linear energy transfer-independent, ${ }^{27-29}$ which is important in proton therapy as linear energy transfer is expected to vary with beam depth. To take this into account in the case of the EBT3 gafchromic films, we applied the corresponding measured quenching correction factors. ${ }^{2}$

Measurements were performed in a water tank (BluePhantom IBA) and in RW3 solid water phantoms. The microDiamond detector was placed inside a BluePhantom scanning system and oriented "in-edge", i.e. perpendicular to the beam to obtain a resolution of $1 \mu \mathrm{m}$ in the spatial fractionation direction of the minibeams. The signal was integrated over a depth of $2.2 \mathrm{~mm}$, corresponding to its diameter, and the scanning step was $100 \mu \mathrm{m}$. The poor resolution in the longitudinal direction $(2.2 \mathrm{~mm})$ made the microdiamond detector unsuitable to measure depth dose curves in pMBRT. Uncertainties were derived from electrometer reproducibility $(1 \%)$, scanning system accuracy $( \pm 0.1 \mathrm{~mm})$, and mean standard deviation in the average dose in the peak and valley regions $(2 \%)$. It should be noted that, even after a low pre-irradiation dose, other authors have also reported a variation of the microDiamond signal stability by more than $1 \%$, which could be due to non-reproducibility between devices at the time of the study. ${ }^{29}$ A conservative overall uncertainty value of $3 \%$ was considered.

PDD curves were only characterised by EBT3 gafchromic films, as they provide the high spatial resolution required for the submillimetric minibeams used. A flatbed scanner (Epson Perfection V750-M Pro Scanner) was used for film readout according to the methodology described in Devic et $\mathrm{al}^{30}$ with a resolution of 1200 dpi. Film handling was performed according to the recommendations of Task Group 55 of the American Association of Physics in Medicine. ${ }^{31}$ Uncertainties in film dose measurements were evaluated according to the method described in Sorriaux et al. ${ }^{32}$ A conservative uncertainty value of $8 \%$ was considered. Scanner readings were calculated by using a home-made $C++$ code using the calibration curves obtained with the optical densities of the irradiated EBT3 gafchromic films.

Comparison of the measurements obtained with the two detectors confirmed the reliability of experimental dosimetry. Finally,

Figure 6. Left: Comparison of PVDR values measured with EBT gafchromic films in a solid water phantom and diamond detector with those simulated by using a fitted GPS virtual source. Right: PDD curves in the central peak measured with EBT gafchromic films and those simulated with the virtual source. GPS, General Particle Source; PDD, percentage depth dose; PVDR, peak-tovalley dose ratio.
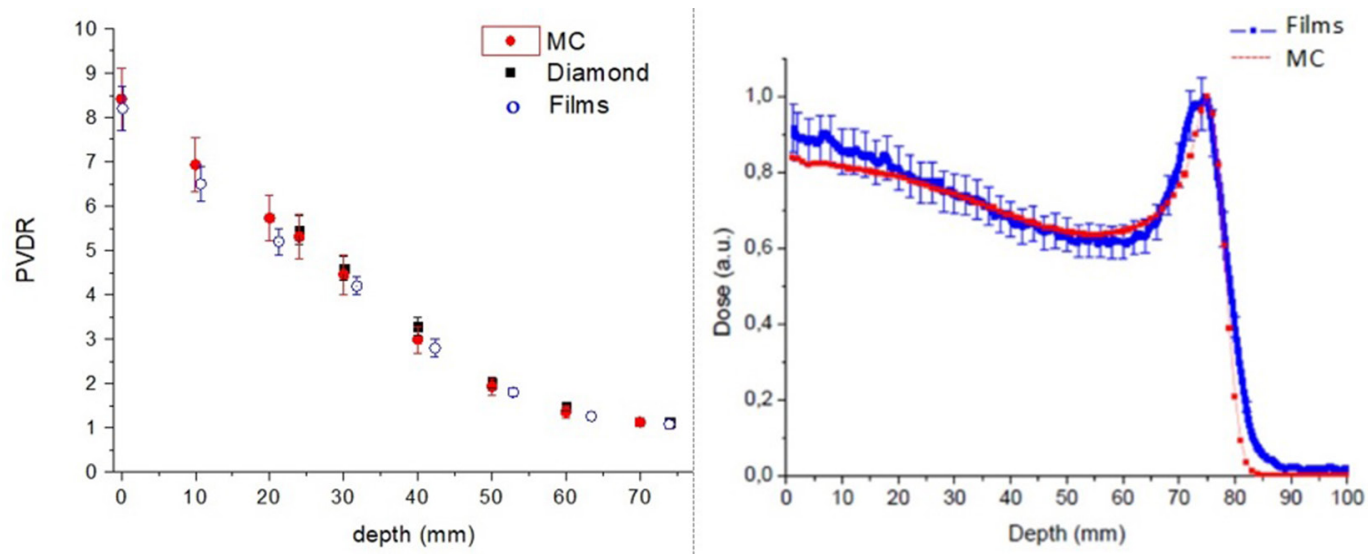
Figure 7. Photograph of a 7-week-old male Fischer rat irradiated at the ICPO Y1-room beamline. The image shows the PMMA in front of the animal's head to target the tumour position when irradiated with $100 \mathrm{MeV}$ protons. ICPO, Institut Curie-Proton therapy centre; PMMA, polymethylmethacrylate.

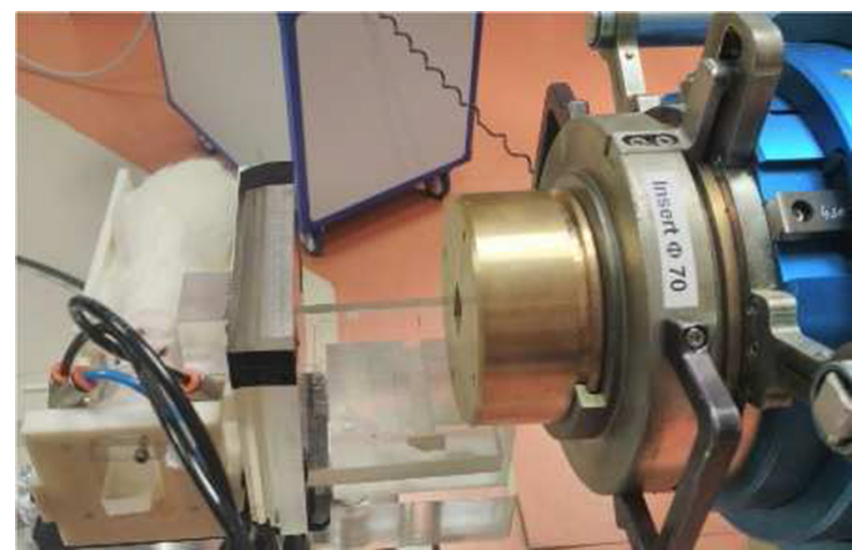

these measurements were used to benchmark our dose calculation engine using the dose calculation acceptability criteria compiled in Technical Report Series 430 (TRS 430). ${ }^{33}$ In particular, the confidence limit $(\Delta)$ defined by Venseelar et $\mathrm{al}^{34}$ was used. See Eq 1.

$$
\Delta=\mid \text { average deviation } \mid+1.5 \mathrm{SD}
$$

SD: standard deviation.

\section{RESULTS}

In this section, we report the dosimetric data acquired, particularly validation of the dose calculation engine developed, with several examples of small animal treatment plans.

\section{Experimental dosimetry}

Figure 3 shows the peak-to-valley dose ratio measured with the two different detectors, which are in good agreement within the uncertainty bars. As expected, multiple Coulomb scattering causes a continuous decrease of the PVDR with increasing depth. Note that the depth dose in water cannot be determined at depths of less than $20 \mathrm{~mm}$ due to the presence of the water tank entrance window.

\section{Benchmarking of the dose calculation engine}

Our Monte Carlo dose calculation engine was validated by comparing experimental and calculated dose distributions. Validation of the whole pMBRT modelling was performed by comparing the results obtained by simulating the Y1-room beamline plus one of the above brass collimators $(0.7 \mathrm{~mm}$ wide with ctc of $3.5 \mathrm{~mm}$ ) over a water phantom with those obtained with EBT3 gafchromic films and diamond detector with 100 $\mathrm{MeV}$ proton irradiations.

Figure 4 (left) shows the PDD curve within the solid water phantom in the central peak of the minibeam array. There is a globally satisfactory agreement for preclinical trials between simulated and measured doses in water (within the uncertainty bars). The discrepancy observed at depths of 55-65 $\mathrm{mm}$ could be due to slight inaccuracies in geometrical modelling of the beamline, which have a greater effect at such small beam sizes. Another cause could be an imperfect multiple coulomb scattering model in the Monte Carlo code.

Similarly, Figure 4 (right) shows the simulated PVDR values versus the experimental PVDR values from the data collected with the PTW diamond detector.

Figure 5 shows a representative lateral profile for a water depth at $24 \mathrm{~mm}$.

$\Delta$ (Eq. 1) complies with the acceptability criteria recommended in TRS $430 .{ }^{33}$ Deviations less than $2 \%$ in the depth dose curves were obtained. A maximum $\Delta$ value of $5 \%$ was found in the high dose/small gradient regions of the profiles of the central peaks. The MC simulated processes are therefore in good agreement with the experimental data, i.e. the Geant $4 \mathrm{MC}$ code with the

Figure 8. PDD (left) and PVDR (right) in the rat brain ( $50 \mathrm{~mm}$ thickness of PMMA, no air gap). Note that the rat brain is about $14 \mathrm{~mm}$ long and the average diameter of the tumour along the beam direction 4 days after implantation (when animals are irradiated) is roughly $4 \mathrm{~mm}$. Since the tumour implantation is approximately on the half of the second brain hemisphere in which the proton beam impinges, i.e. at a depth of approximately $10.5 \mathrm{~mm}$ of the rat brain, the tumour volume extends approximately from 8.5 to $12.5 \mathrm{~mm}$. Note that the preferential direction of tumour growth is unpredictable. PDD, percentage depth dose; PMMA, polymethylmethacrylate; PVDR, peak-to-valley dose ratio.
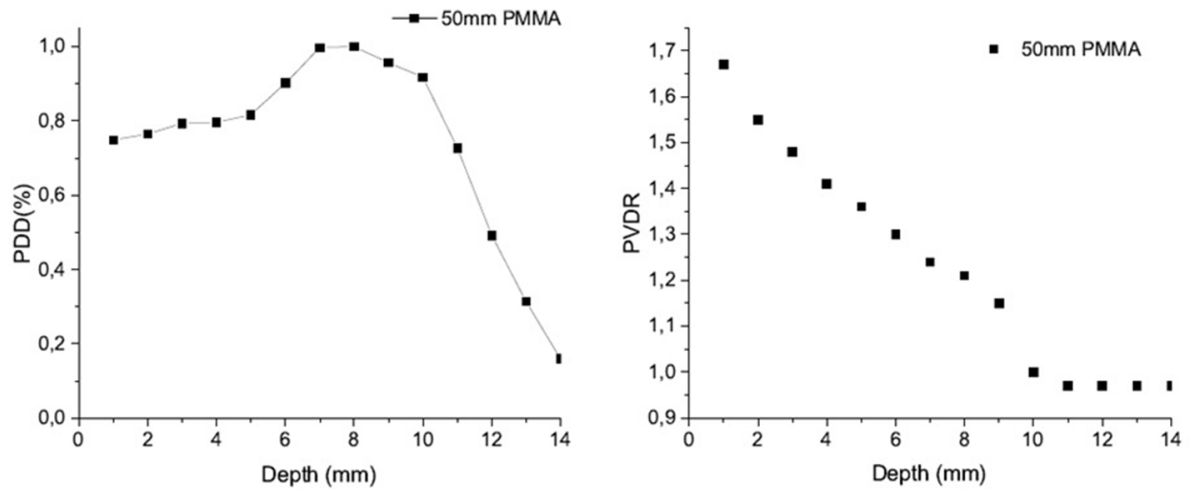
Figure 9. Coronal 2D dose distributions on computed tomography images of 7-week-old Fischer 344 rats. From left to right, they correspond to broad beam (conventional) irradiation and proton minibeam irradiations targeting the tumour and crossing the rat head, respectively. ${ }^{37,38}$ Black and red lines indicate the approximate position of the centre of the tumour.
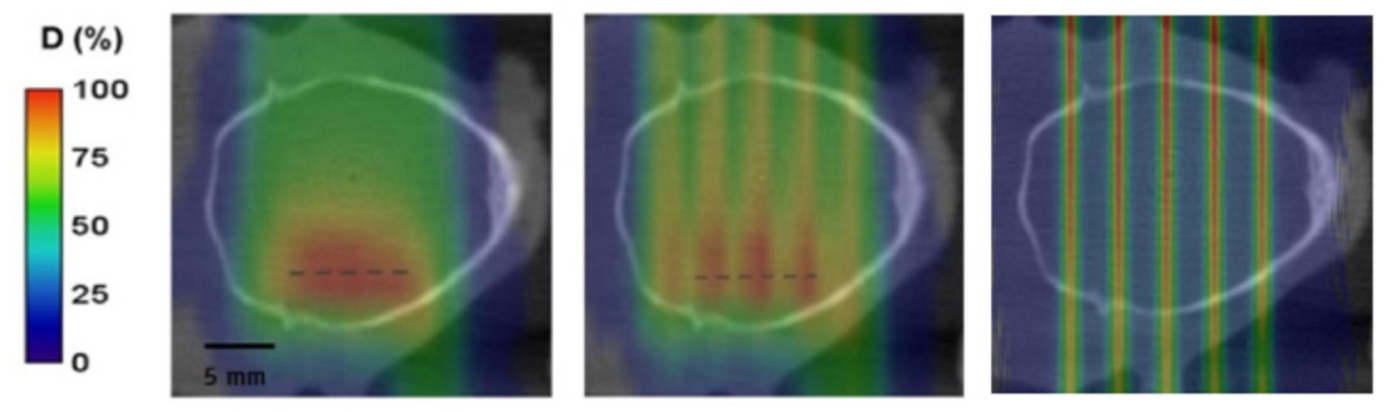

physics list recommended by GATE collaboration for proton therapy appears to accurately simulate the multiple coulomb scattering process for the particular conditions of small fields.

Independent Phase Space Files were scored at the nozzle exit (Figure 1) by means of the Phase Space GATE-actor ${ }^{8}$ that tallies information about particles entering the volume to which the actor is attached. They were scored as ROOT data analysis format. ${ }^{35}$ On the basis of these beam optics parameters, the beam can be characterised at that depth (nozzle exit). Fitting was performed by converting raw data to ROOT two-dimensional histograms. The energy spread was characterised by a Gaussian standard deviation. These beam features were used to model a virtual source based on the predefined General Particle Source. As explained in "Simulationof one passive scattering beamline at ICPO".

Iterative comparison of the simulated and measured dose distributions was performed for fine tuning of the parameters of these sources. Figure 6 (left) shows the final best fitted General Particle Source-virtual source with the experimental data with a divergence of $4.5 \mathrm{mrad}$ in agreement with previous values. ${ }^{18}$ The source-to-phantom distance was considered to $100 \mathrm{~cm}$, as it was demonstrated in Cécile ${ }^{16}$ that the peak-to-entrance ratio becomes constant. A Gaussian spread was created by using a two-dimensional beam travelling along the local negative $\mathrm{z}$ axis.

Figure 10. Coronal 2D dose distributions on computed tomography images of 7-week-old Fischer 344 rats for ctc of $3 \mathrm{~mm}$ (left) and $2.8 \mathrm{~mm}$ (right). A smaller ctc was associated with greater homogeneity of the dose distribution over the tumour. Black lines indicate the approximate position of the centre of the tumour. 2D, two-dimensional.
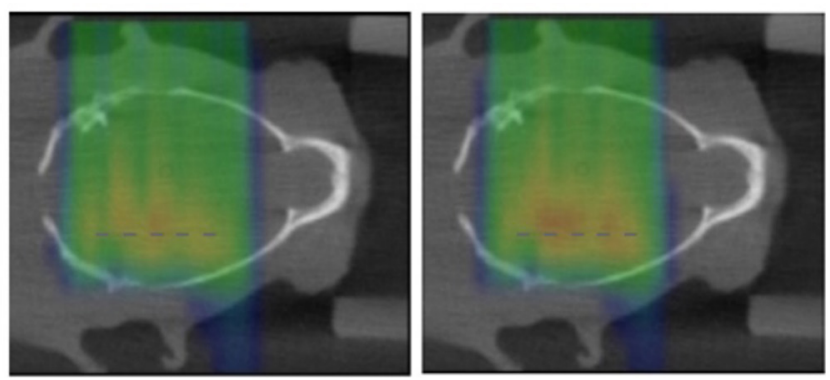

Similarly, Figure 6 (right) shows the PDD curve in the central peak of the minibeam array. Both figures show good agreement between experimental and simulated results. A minor discrepancy at a depth of $0 \mathrm{~cm}$ was found, which could be due to minor inaccuracies in phantom alignment or phantom positioning or to the uncertainties in the energy spread used in the virtual source.

On the basis of these results and to speed-up simulations, forthcoming simulations were performed with that virtual source.

Note that the influence of the uncertainty of the ionisation potential on the proton range is out of the scope of this study. Nevertheless, although we used the GATE recommendation value for ionisation potential of the water (i.e. $75 \mathrm{eV}$ ), we corroborated that there is not significant differences in the PDD for a $100 \mathrm{MeV}$ proton energy by using the current ICRU73 recommended one (i.e. $78 \mathrm{eV}$ ) with the virtual source described in "Virtual source modelling". Further studies regarding the influence of various sets of stopping power data with I values for water between 67 and $80 \mathrm{eV}$ can be found in Luhr et al. ${ }^{36}$ They found deviations of the order of $1 \%$ in the plateau region or range in water for that I value range.

\section{Dosimetry for in vivo experiments: rat brain PMBRT irradiations}

Two types of experiments were performed: animals were irradiated with the Bragg peak located at the tumour position and animals were irradiated in the plateau region. As the primary objective was to optimally reproduce clinically relevant conditions, a clinically relevant energy (i.e. $100 \mathrm{MeV}$ ) was used. Some attenuation material is required to place the Bragg peak in the tumour. Polymethylmethacrylate (PMMA) was used (Figure 7). The required thickness was calculated by Monte Carlo simulations taking into account irradiations of 7 week-old Fischer rats. We used a $0.4 \mathrm{~mm}$ wide brass collimator with a ctc of $3.2 \mathrm{~mm}$, as it delivers a homogeneous dose distribution in water. ${ }^{21}$

Figure 8 shows the optimal value for PDD and PVDR inside the rat brain with no air gap between the rat head and PMMA slabs. Note that the rat brain is approximately $14 \mathrm{~mm}$ along on a midline section. Since the site of tumour implantation is approximately on the half of the second brain hemisphere in which the proton beam impinges, i.e. at a depth of approximately $10.5 \mathrm{~mm}$ 
of the rat brain, the optimal PMMA thickness is between 50 and $48 \mathrm{~mm}$. As the FWHM of the Bragg curve at that depth is approximately $6 \mathrm{~mm}$, the tumour sizes considered (see materials and methods section) would be totally covered.

Figure 9 shows the dose profiles at the middle of the brain and the central part of the tumour for the two cases considered above. The PVDR ratios were $1.20 \pm 0.05$ and $6.1 \pm 0.2$, respectively. ${ }^{37,38}$

In the first case, with a ctc of $3.2 \mathrm{~mm}$ ctc, a homogeneous dose distribution inside the rat brain is not completely obtained due to the fact that the morphology and composition of the animal's head both differ from the water archetype. A homogeneous dose distribution in the tumour position can be achieved by reducing the centre-to-centre distance of the minibeams. For example, as shown in Figure 10, dose distributions become more homogeneous in the tumour position when a smaller ctc is considered, ranging from 3 to $2.8 \mathrm{~mm}$.

These results illustrate the importance of reliable calculations to interpret results. For example, as those performed for understanding dose distributions inside the rat head in preclinical trials. ${ }^{37,38}$ In particular, the dose calculation engine for pMBRT implementation was used to guide the first two critical preclinical studies. We first compared animal survival when animals were irradiated with pMBRT and standard proton therapy in an in vivo test with RG2 glioma-bearing rats. In this study, we evaluated the efficacy of pMBRT for the treatment of highgrade gliomas and, although some spatial fractionation was still observed at the Bragg position, tumour eradication was observed in $67 \%$ of the animals treated by pMBRT. ${ }^{37}$ We then reported the

Figure 11. Dose distribution on CT scans of the rat head irradiated with $50 \mathrm{MeV}$ (left) and $70 \mathrm{MeV}$ (right) proton minibeams. Spatial fractionation was observed in both cases.
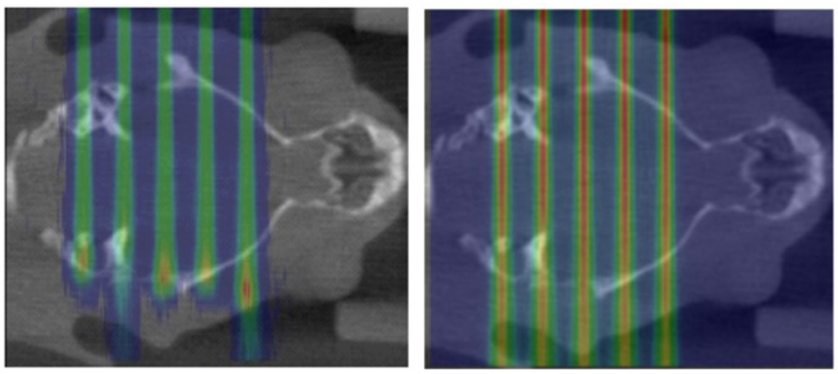

first evaluation of tumour control efficacy in glioma-bearing rats with highly spatially modulated proton beams, i.e. large areas of the tumour received low doses (valley doses). In this case, we simulated the lateral irradiation of rat brain in the plateau region with a dose rate of $2 \mathrm{~Gy} / \mathrm{min}$ at a depth of $1 \mathrm{~cm}$. We showed that the heterogeneous dose distribution achieved significant tumour control in $22 \%$ of cases. ${ }^{38}$

Simulations with 50 and $70 \mathrm{MeV}$ protons, with ranges in water of approximately 2.3 and $4.1 \mathrm{~cm}$, respectively, were also performed to compare these results with those obtained with usual preclinical energies. Figure 11 shows the dose distributions in the same intermediate axial plane when animals were irradiated with 50 and $70 \mathrm{MeV}$ proton minibeams.

PVDRs in the tumour position were $10.4 \pm 0.6$ and $7.3 \pm 0.4$ for the 50 and $70 \mathrm{MeV}$ proton irradiations, respectively. PVDRs were higher for low energies due to less homogeneous dose distribution, resulting in an increase of $\Delta=4.3 \pm 0.8$ and $1.2 \pm 0.6$ over the PVDR compared to the PVDR with $100 \mathrm{MeV}$ pMBRT.

\section{CONCLUSIONS}

This paper reports on the development and experimental validation of a dose calculation engine for pMBRT. The small beam sizes used in this study makes experimental and theoretical dosimetry particularly challenging. An overall good agreement between experimental and calculations was obtained, although further evaluations of the performance of the multiple coulomb scattering models implemented in the codes is needed and will be the subject of future works. This engine can be used to correlate the results on ongoing preclinical studies at the Orsay proton therapy centre with the dose distributions. In addition, it will be useful to design forthcoming experiments. To the best of our knowledge, this is the first dose calculation engine developed for pMBRT.

\section{ACKNOWLEDGMENT}

This project has received funding from the European Union's Horizon 2020 research and innovation programme under the Marie Skłodowska-Curie grant agreement No 74,5109. Calculation time was granted at Castilla y León Supercomputing Center (Calendula) from the national RES access programme (Spanish Supercomputing Network Area) and the MareNostrum Barcelona Supercomputing Center from the Partnership for Advanced Computing in Europe (PRACE Project Access Call 14th and $16^{\text {th }}$, proposals number 2016153507 and 2017174196 , respectively).

\section{REFERENCES}

1. Prezado Y, Fois GR. Proton-minibeam radiation therapy: a proof of concept. Med Phys 2013; 40: 031712. doi: https://doi.org/ $10.1118 / 1.4791648$

2. Peucelle C, Nauraye C, Patriarca A, Hierso E, Fournier-Bidoz N, Martínez-Rovira I, et al. Proton minibeam radiation therapy: experimental dosimetry evaluation. Med Phys 2015; 42: 7108-13. doi: https://doi.org/ $10.1118 / 1.4935868$

3. De Marzi L, Patriarca A, Nauraye C, Hierso E, Dendale R, Guardiola C, et al. Implementation of planar proton minibeam radiation therapy using a pencil beam scanning system: a proof of concept study. Med Phys 2018; 45: 5305-16. doi: https://doi. org/10.1002/mp.13209

4. Prezado Y, Jouvion G, Hardy D, Patriarca A, Nauraye C, Bergs J, et al. Proton minibeam radiation therapy spares normal rat brain: long-term clinical, radiological and 
histopathological analysis. Sci Rep 2017; 7: 14403. doi: https://doi.org/10.1038/s41598017-14786-y

5. Girst S, Greubel C, Reindl J, Siebenwirth C, Zlobinskaya O, Walsh DWM, et al. Proton Minibeam Radiation Therapy Reduces Side Effects in an In Vivo Mouse Ear Model. Int J Radiat Oncol Biol Phys 2016; 95: 234-41. doi: https://doi.org/10.1016/j.ijrobp.2015.10.020

6. Prezado Y, Jouvion G, Guardiola C, Gonzalez W, Juchaux M, Bergs J, et al. Tumor control in RG2 Glioma-Bearing rats: a comparison between proton Minibeam therapy and standard proton therapy. Int $J$ Radiat Oncol Biol Phys 2019; 104: 266-71. doi: https://doi. org/10.1016/j.ijrobp.2019.01.080

7. Prezado Y, Jouvion G, Patriarca A, Nauraye C, Guardiola C, Juchaux M, et al. Proton minibeam radiation therapy widens the therapeutic index for high-grade gliomas. Sci Rep 2018; 8: 16479. doi: https://doi.org/10. 1038/s41598-018-34796-8

8. www.opengatecollaboration.org

9. GEANT4 Collaboration. GEANT4 (geometry and tracking) code.

10. Agostinelli S, Allison J, Amako K, Apostolakis J, Araujo H, Arce P, et al. Geant4-a simulation toolkit. Nuclear Instruments and Methods in Physics Research Section A: Accelerators, Spectrometers, Detectors and Associated Equipment 2003; 506: 250-303. doi: https://doi.org/10.1016/ S0168-9002(03)01368-8

11. Allison J, Amako K, Apostolakis J, Araujo $\mathrm{H}$, Arce Dubois P, Asai M, et al. Geant4 developments and applications. IEEE Transactions on Nuclear Science 2006; 53: 270-8. doi: https://doi.org/10.1109/TNS. 2006.869826

12. http://geant4.in2p3.fr/IMG/pdf_PhysicsLists. pdf

13. http://geant4.web.cern.ch/geant4/support/ proc_mod_catalog/physics_lists/referencePL. shtml

14. Grevillot L, Frisson T, Zahra N, Bertrand D, Stichelbaut F, Freud N, Sarrut D, et al. Optimization of GEANT4 settings for proton pencil beam scanning simulations using gate. Nuclear Instruments and Methods in Physics Research Section B: Beam Interactions with Materials and Atoms 2010; 268: 3295-305. doi: https://doi.org/10.1016/j.nimb.2010.07.011

15. Seravalli E, Robert C, Bauer J, Stichelbaut F, Kurz C, Smeets J, et al. Monte Carlo calculations of positron emitter yields in proton radiotherapy. Phys Med Biol 2012; 57: 1659-73. doi: https://doi.org/10.1088/00319155/57/6/1659

16. Cécile P. Spatial fractionation of the dose in charged particle therapy. $\mathrm{PhD}$ thesis. Univ. Paris-Saclay; 2016.
17. Patriarca A, Fouillade C, Auger M, Martin F, Pouzoulet F, Nauraye C, et al. Experimental set-up for flash proton irradiation of small animals using a clinical system. Int J Radiat Oncol Biol Phys 2018; 102: 619-26. doi: https://doi.org/10.1016/j.ijrobp.2018.06.403

18. Stankovskiy A, Kerhoas-Cavata S, Ferrand R, Nauraye C, Demarzi L. Monte Carlo modelling of the treatment line of the proton therapy center in Orsay. Phys Med Biol 2009; 54: 2377-94. doi: https://doi.org/10.1088/ 0031-9155/54/8/008

19. Proton therapy physics. CRC press editions 2012; Harad Paganetti.

20. Seco J, Verhaegen F. Monte Carlo Techniques in Radiation Therapy: CRC Press editions; 2013.

21. Guardiola C, Peucelle C, Prezado Y. Optimization of the mechanical collimation for minibeam generation in proton minibeam radiation therapy. Med Phys 2017; 44: 1470-8. doi: https://doi.org/10.1002/mp. 12131

22. Chetty IJ, Rosu M, Kessler ML, Fraass BA, Ten Haken RK, Kong Feng-Ming (Spring), Ten H, Kong FM, et al. Reporting and analyzing statistical uncertainties in Monte Carlo-based treatment planning. Int $J$ Radiat Oncol Biol Phys 2006; 65: 1249-59. doi: https://doi.org/10.1016/j.ijrobp.2006.03. 039

23. Gualdrini G, Mariotti F, Wach S, Bilski P, Denoziere M, Daures J, et al. A new cylindrical phantom for eye lens dosimetry development. Radiat Meas 2011; 46: 1231-4. doi: https://doi.org/10.1016/j.radmeas.2011. 08.025

24. Lansonneur P, Favaudon V, Heinrich S, Fouillade C, Verrelle P, De Marzi L. Simulation and experimental validation of a prototype electron beam linear accelerator for preclinical studies. Physica Medica 2019; 60: 50-7. doi: https://doi.org/10.1016/j.ejmp. 2019.03.016

25. Gafchromic EBT3 dosimetry media. www. ashland.com. 2016.

26. PTW microDiamond, synthetic diamond detector specifications. www.ptw.de, 2016.

27. Mandapaka AK, Ghebremedhin A, Patyal B, Marinelli M, Prestopino G, Verona C, et al. Evaluation of the dosimetric properties of a synthetic single crystal diamond detector in high energy clinical proton beams. Med Phys 2013; 40: 121702121702-9vol.. doi: https:// doi.org/10.1118/1.4828777

28. Rossomme S, Denis JM, Souris K, Delor A, Bartier F, Dumont D, et al. Let dependence of the response of a PTW-60019 microDiamond detector in a $62 \mathrm{MeV}$ proton beam. Phys Med 2016; 32: 1135-8. doi: https://doi.org/10.1016/j.ejmp.2016.08.017
29. Marsolat F, De Marzi L, Patriarca A, Nauraye C, Moignier C, Pomorski M, et al. Dosimetric characteristics of four PTW microDiamond detectors in high-energy proton beams. Phys Med Biol 2016; 61: 6413-29. doi: https://doi.org/10.1088/00319155/61/17/6413

30. Devic S, Seuntjens J, Sham E, Podgorsak EB, Schmidtlein CR, Kirov AS, et al. Precise radiochromic film dosimetry using a flatbed document scanner. Med Phys 2005; 32(7Part1): 2245-53. doi: https://doi.org/10. 1118/1.1929253

31. Niroomand-Rad A, Blackwell CR, Coursey BM, Gall KP, Galvin JM, McLaughlin WL, et al. Radiochromic film dosimetry: recommendations of AAPM radiation therapy Committee task group 55. Med Phys 1998; 25: 2093-115. doi: https://doi.org/10. 1118/1.598407

32. Sorriaux J, Kacperek A, Rossomme S, Lee JA, Bertrand D, Vynckier S, et al. Evaluation of Gafchromic ${ }^{\circledR}$ EBT3 films characteristics in therapy photon, electron and proton beams. Physica Medica 2013; 29: 599-606. doi: https://doi.org/10.1016/j.ejmp.2012.10.001

33. International Atomic Energy Agency Commissioning and quality assurance of computerized planning systems for radiation treatment of cancer. Technical Report Series No. 430, IAEA, Vienna 2004;.

34. Venselaar J, Welleweerd H, Mijnheer B, et al. Tolerances for the accuracy of photon beam dose calculations of treatment planning systems. Radiotherapy and Oncology 2001; 60: 191-201. doi: https://doi.org/10.1016/ S0167-8140(01)00377-2

35. Brun R, Rademakers F. ROOT-An object oriented data analysis framework, proceedings AIHENP'96 workshop, Lausanne. Nucl Inst \& Meth in Phys Res A 1996; 389: 81-6.

36. Lühr A, Hansen DC, Jäkel O, Sobolevsky N, Bassler N. Analytical expressions for waterto-air stopping-power ratios relevant for accurate dosimetry in particle therapy. Phys Med Biol 2011; 56: 2515-33. doi: https://doi. org/10.1088/0031-9155/56/8/012

37. Prezado Y, Jouvion G, Guardiola C, Gonzalez W, Juchaux M, Bergs J, et al. Tumor control in RG2 Glioma-Bearing rats: a comparison between proton Minibeam therapy and standard proton therapy. Int $J$ Radiat Oncol Biol Phys 2019; 104: 266-71. doi: https://doi. org/10.1016/j.ijrobp.2019.01.080

38. Prezado Y, Jouvion G, Patriarca A, Nauraye C, Guardiola C, Juchaux M, et al. Proton minibeam radiation therapy widens the therapeutic index for high-grade gliomas. Sci Rep 2018; 8: 16479. doi: https://doi.org/10. 1038/s41598-018-34796-8 